\title{
Behavior of Certain Wavelets in Classification of Orthopaedic Images of Different Modalities
}

\author{
M. V. Latte ${ }^{1 *}$ Kumar Swamy. $V^{2}$ and B.S.Anami ${ }^{3}$ \\ ${ }^{1}$ Principal, JSSATE, Bangalore, \\ ${ }^{2}$ Asst Professor, Department of EEE, KLEIT, Hubli, \\ ${ }^{3}$ Principal, KLEIT, Hubli \\ mvlatte@rediffmail.com,swamy_bvbdigital@yahoo.co.in, \\ anami_basu@hotmail.com
}

\begin{abstract}
Orthopedicians often identify imaging modality visually out of their experience. To be effective, the process needs to be automated. This paper presents a behavior of wavelets in classification of orthopedic imaging modalities using Artificial Neural Network (ANN). In this work, we have considered orthopedic imaging modalities, namely, X-ray, CT and MRI and Bone scan images. Four wavelets, namely Haar, Daubechies, Symlets and Coiflets are used for sub band decomposition and their approximation co-efficients are recorded. Features, namely, mean standard deviation, median, variance and entropy is drawn from the decomposed images. Results are drawn from the performance of these wavelets at five levels of decomposition. Feature reduction is based on the classification accuracies which are analysed using wavelets. The experimental results show that the proposed method achieves satisfactory results with an average accuracy of $98 \%$ for four wavelets and for all the modalities considered. The study can be extended to include other modalities in medical field. The work is useful for orthopaedics practitioners.
\end{abstract}

Keywords: Wavelets, Image modalities, Medical images, x-ray images, Image processing

\section{Introduction}

Technology plays a vital role in all the areas wherever it is applicable, particularly medical healthcare domain. In this domain, technology is focused on a term called medical imaging. Medical imaging equipments are built using computer vision and image processing techniques such as X-Ray, CT scan and MRI and the like. In hospitals, normally more casualties are in orthopedic department where musculoskeletal trauma, sports injuries and fractures are diagnosed. Orthopeadician popularly uses four imaging technologies, namely, X-ray, CT, MRI and Bone scans to diagnose the severity of the fractures, malignant tumours, bone cancer and so on. Some images of bone taken from four orthopedic imaging modalities are shown in Figure 1.
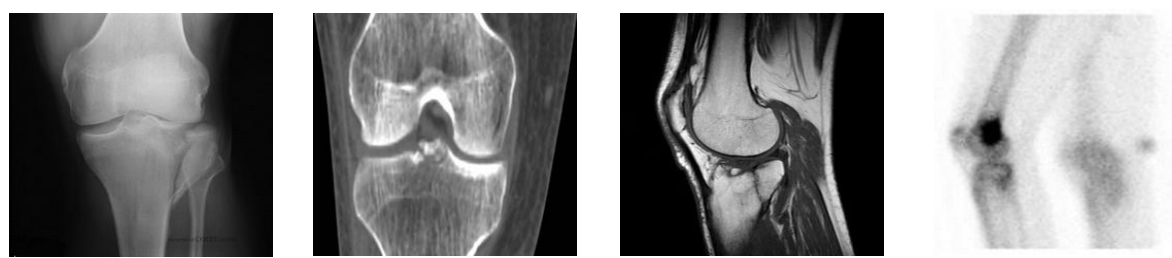

Figure 1. Typical Images of Bone (a) X-Ray Image (b) CT Scan (c) MRI Scan (d) Bone Scan

${ }^{*}$ Corresponding Author 
As radiologist identify the given image is of x-ray machine or CT or MRI or Bone scan out of their experience. There is a need for automation to identify these modalities which are popularly used by almost all the orthopeadician. Hence, we propose a methodology on classification of orthopedic imaging modalities using wavelets.

Automation is carried based on the inputs given from radiologist. They (radiologist) identify the modality as X-Ray, CT, MRI and bone scan based on the following factors. $\mathrm{X}$-ray can be identified as only bone is exposed as brighter and skin has a bit dull in brightness as shown in Figure 1.a, X-ray are advised when orthopeadician suspects a fracture. CT scan modality can be identified based on some more information available when compare with x-ray such as organs, tissues or tumors as shown in Figure.1.b, MRI scan are advised to see the torn ligament and cartilage as shown in Figure.1.c, Bone scan are advised to view the spreading of bone cancer as shown in Figure.1.d, Figure.1.a, b, c and $\mathrm{d}$, are different modality shown because the variation of information seen with the same body part (knee).

Proposed methodology involves image acquisition, preprocessing, wavelets, level selection, feature selection and a classifier. Input images are acquired from various hospitals. Wavelets are used to decompose the image and draw its approximation coefficients and features like mean, standard deviation, median, variance and entropy are computed to form the feature set. Orthopedic imaging modalities contain frequency variations which can be extracted and seen through wavelets. Although Fourier transform is probably the most popular transform being used (especially in electrical engineering), it is not the only one. There are many other transforms like Hilbert transform, short-time Fourier transform, Wigner distributions, the Radon Transform, and of course our featured transformation, the wavelet transform, constitute only a small portion of a huge list of transforms that are available at engineer's and mathematician's disposal. Every transformation technique has its own area of application, with advantages and disadvantages, and the wavelet transform (WT) is no exception. In signal processing, wavelets make it possible to recover weak signals from noise. This has proven useful especially in the processing of X-ray and magnetic-resonance images in medical applications. Images processed in this way can be "cleaned up" without blurring or muddling the details. Often times, the information that cannot be readily seen in the timedomain can be seen in the frequency domain. The discrete wavelet transform (DWT), on the other hand, provides sufficient information both for analysis and synthesis of the original signal, with a significant reduction in the computation time. Wavelets can also be constructed with rough edges, to better approximate real-world signals. Wavelets families, namely, Haar, Daubechies, Coiflets and Symlets are used for the work. Feature reduction technique is done based on their performances to identify the modalities using ANN.

\subsection{Literature Survey}

The literature survey is carried out to know the state-of-the-art in the related areas. To the best of our knowledge, few researchers have carried the work on modality classification using wavelets focusing on content based image retrieval (CBIR) but negligible amount of work is reported for bone image modality classification using wavelets specifically on wavelets families, namely, Haar, Daubechies, Symlets and Coiflets. Hence, the proposed work. The studied literature is organized into two perspectives: pattern recognition using wavelets as features and the other is modality classification using different image processing approach. Accordingly, the following works are cited.

Features were extracted from the wavelet coefficients of measured process signals which are eventually calculated by the Euclidean norms of the clusters. It was used in recognizing different kinds of lung sounds for diagnosis of pulmonary diseases [1]. An affine-invariant image retrieval approach based on wavelet detector was proposed which used space-tree property of the transform coefficients to estimate the interest points [2]. 
Combination of Wavelet-based features and SIFT features were used to extract patches from images, and then PCA transformation was used to reduce the dimensionality of the feature vectors. The reduced vectors are used to train Gaussian Mixture Models (GMMs) in which the mixture weights and Gaussian parameters are updated iteratively [3]. Wavelet features were computed for every patch that was extracted over the salient points taken from the original image. Thus extracted features are trained in order to get a learning model, tested and classified using SVM [4]. Wavelet moment invariants together with a discriminative feature selection method were used for the classification of seemingly similar objects. Using a minimum-distance classifier, wavelet moment invariants achieved the highest classification [6].

A robust text localization approach was used which detects horizontally aligned text with different sizes, fonts, colors and languages. K-means algorithm was used to classify text areas in the image [8]. Have proposed segmentation pipeline for computer-based automatic analysis of multi-modal tomograpic images. The segmentation pipeline includes texture analysis, classification with a modified Kohonen feature Map [9]. Have given a novel technique to extract features for characterization and segmentation of texture at multiple scales based on block by block comparison of wavelet co occurrence [10]. Energy information obtained from wavelet transform for classification of medical images according to imaging modalities and body parts. They have used two types of wavelets and showed that energy obtained in either case is quite distinct for each of the body part [11].

Semantically based set of visual features, their relevance and organization for capturing the semantics of different imaging modalities was proposed. The features used in conjunction with a new categorization metric enabling "intelligent" annotation for searching of medical databases [12]. In each level of the hierarchical classifier, using a new merging scheme and multilayer perceptron (MLP) classifier (merging-based classification), homogenous (semantic) classes were created from over lapping classes in the database. Merging scheme employs three measures to detect the overlapping classes: accuracy, mis-classified ratio, and dissimilarity [15]. A frame work consisting of machine learning methods for image pre-filtering, similarity matching using statistical distance measures, and a relevance feedback (RF) scheme were proposed [17].

Continuous and probabilistic image representation scheme using Gaussian mixture modeling (GMM) along with information - theoretic image matching via the KullbackLeibler (KL) measure was proposed [18]. Multi-disciplinary approach to tackle the classification problem by combining image features, meta-data, textual and referential information is given. System's accuracy was tested on the ImageCLEF2011 medical modality classification dataset [19]. A hybrid meta-heuristic swarm Intelligence-based search technique, called mixed gravitational search algorithm (MGSA), was employed. Some feature extraction parameters (i.e., the parameters of a 6-tap parameterized orthogonal mother wavelet in texture features and quantization levels in color histogram) are optimized to reach a maximum precision of the CBIR systems [21]. Have given local patch for representation of the image content and a bag-of-features, to categories the image with a kernel based SVM classifier [22].

Table 1 gives the summary of the literature in terms of features, classifiers and their performance. To the best of our knowledge, no reported work is attempted to classify orthopedic images from different modalities based on wavelets. 
Table 1. Summary of the Literature Survey

\begin{tabular}{|c|c|c|c|}
\hline Reference & Features & Classifier & Accuracy \\
\hline 1 & Fast wavelet transform & BPNN & $100 \%$ \\
\hline 2 & Wavelet interest points & Affine invariant & -NA- \\
\hline 3 & Combined wavelet and SIFT & Gaussian mixture model & -NA- \\
\hline 4 & 2-Dimensional gabor wavelet & Linear SVM & $80 \%$ \\
\hline 5 & Wavelet moment variants & & $85-90 \%$ \\
\hline 6 & Wavelet moment variants & Minimum distance & $100 \%$ \\
\hline 7 & DWT & $\begin{array}{l}\text { Progressive texture class } \\
\text { algorithm. }\end{array}$ & $100 \%$ \\
\hline 8 & DWT & K-NN. & $90 \%$ \\
\hline 9 & DWT & Modified Kohonen & $96 \%$ \\
\hline 10 & DWT & Co-occurrence & -NA- \\
\hline 15 & Shape and texture & Multi level perceptron & $90.83 \%$ \\
\hline 16 & Local gradient & Gaussian kernel & $92 \%$ \\
\hline 17 & Color, texture and edge & SVM & -NA- \\
\hline 18 & $\begin{array}{lll}\begin{array}{l}\text { Intensity, } \\
\text { information }\end{array} & \text { texture, spatial } \\
\end{array}$ & GMM-KL measure. & $97.5 \%$ \\
\hline 19 & $\begin{array}{l}\text { Textual and referential } \\
\text { information }\end{array}$ & Linear SVM & $88.47 \%$ \\
\hline 20 & Text & SVM & $94 \%$ \\
\hline 22 & $\begin{array}{l}\text { Local patch representation, } \\
\text { content and spatial }\end{array}$ & SVM & $94 \%$ \\
\hline 24 & Mean and variance & Similarity measure & $97 \%$ \\
\hline
\end{tabular}

Legend: BPNN - Back propagation neural network; SIFT - Scale-invariant feature transform, DWT - Discrete wavelet transform; k-NN - k-Nearest neighbor classifier; SVM - Support vector machine, GMM-KL - Gaussian mixture modeling KullbackLeibler(KL). N/A - Not available

From the literature survey, it is observed that researchers have worked on medical image classification based on multimodality. The content, graphical and decision based retrieval of information is carried out. However, no work on classification of medical image modalities, more so on the orthopedic images is observed. Hence, it is the motivation for the present work.

The paper is organized into four sections. Section two contains the proposed methodology. The modality classification and results are given in section three. Section four gives conclusion of the work

\section{Methodology Proposed}

Input images are acquired from various hospital and laboratories to create the database required for the work. As the input images is porn to have film noise. Different noise removal filters are applied and their PSNR values are computed and compared. For the work carried, weiner filtering gave highest PSNR vales. Wavelet family, namely, Haar, Daubechies, Symlets and Coiflets are used for sub-band decomposition of processed images. Decomposition is done for five levels, 3rd level of decomposition gave the better results and is finalized to carry further work. Wavelet approximation is computed from wavelet family individually and five features, namely, mean, standard deviation, median, variance and entropy are drawn. Performance are studied and plotted by considering indiviual feature, group of two features, group of three features and all the five features 
together. Overview of the methodology is depicted in Figure.2, It comprises of six stages, namely, image acquisition, pre-processing, wavelets, level selection, feature selection and classification.

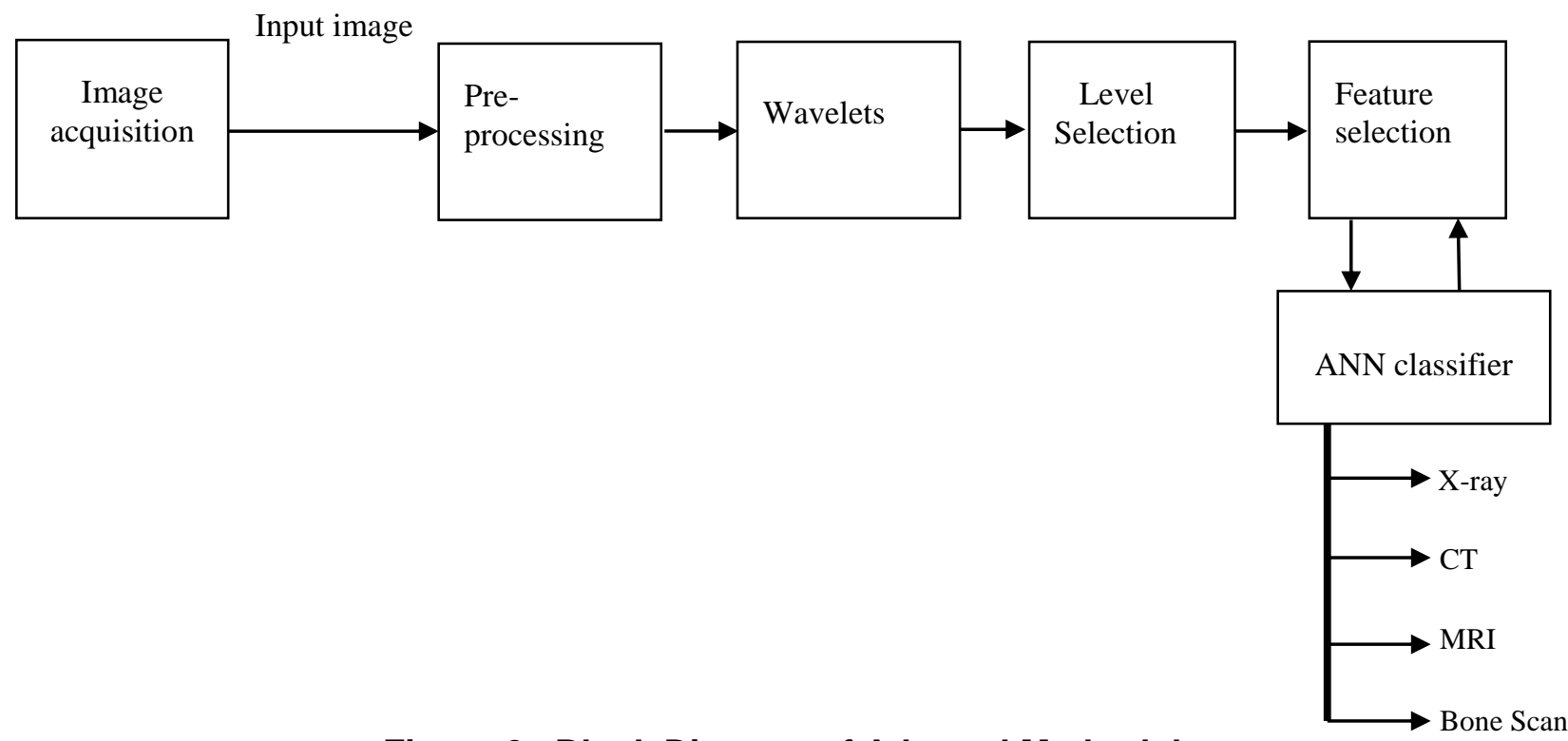

Figure 2. Block Diagram of Adopted Methodology

The individual features have given unsatisfactory results. We have tried the combination of two; three and four features and experimented. The back propagation neural network classifier is employed for modality classification.

2.0.1. Image Acquisition: The $X$-ray, $C T$, $M R I$ and Bone scan images are acquired from different hospitals and laboratories. A total of 2000 image were considered for the proposed work. The x-ray images are obtained from machine "Essenta DR Compact", a product of Philips and "Agfa CR-35X", a product of Agfa healthcare. X-ray images of knee part of leg are shown in Figure.3 (a), (b) and (c). The CT scan images are shown in Figure.4 (a), (b) and (c), and MRI images in Figure.5 (a), (b) and (c). Bone scan images in Figure.6 (a), (b) and (c) In particular only knee part is considered, as it is the only that part of the body is subjected to x-ray, CT and MRI imaging as consulted by orthopeadicians.

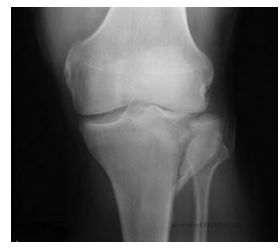

(a)

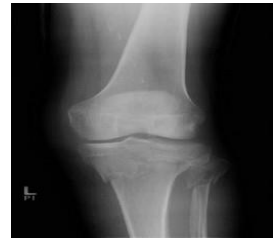

(b)

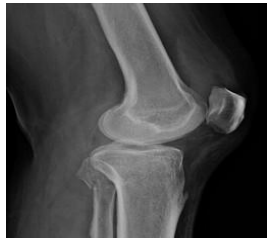

(c)

Figure 3. Typical X-Ray Images

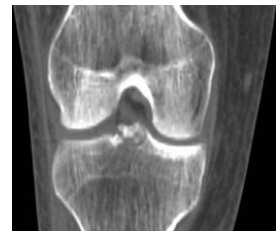

(a)

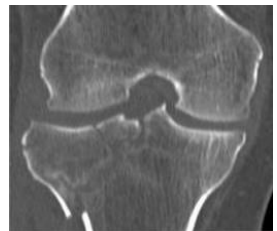

(b)

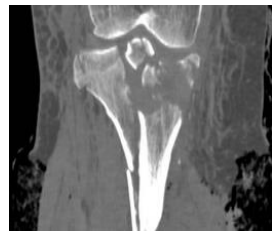

(c)

Figure 4. Typical CT Scan Images 


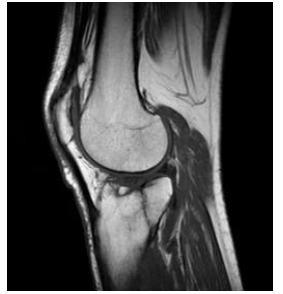

(a)

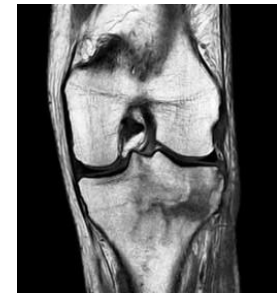

(b)

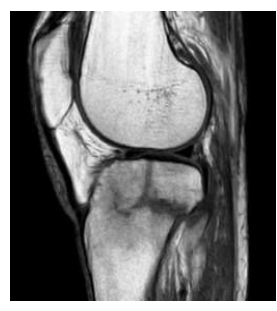

(c)

Figure 5. Typical MRI Scan Images

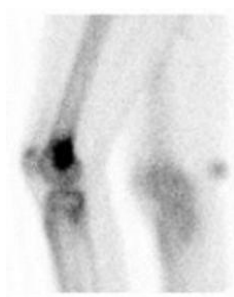

(a)

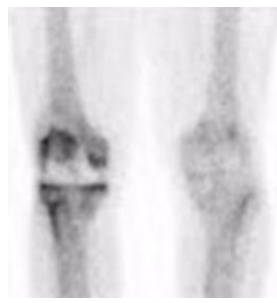

(b)

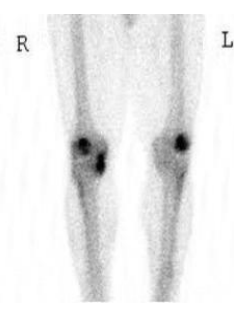

(c)

Figure 6. Typical Bone Scan Images

2.0.2. Preprocessing: In this stage, input images are made eligible to extract features for further experimentation. It involves application of noise removal filters. We have used weiner, mean and median filtering techniques for the work. Mean Square Error (MSE), Root Mean Square Error (RMSE), Peak signal to noise ratio (PSNR) values is calculated from the images. Results prove Weiner filtering performs better when compared with other filtering techniques for orthopedic imaging modalities considered. To understand the work flow, we are showing the values calculated for only 8 images. The proposed system does not support for analog X-ray images as it contains film noise. Also poor film development reduces the quality of the image which at times needs to repeat X-ray. In this work we have considered digital $\mathrm{x}$-ray as the cost is low when compared to analog $\mathrm{X}$-ray images. MSE, RMSE, PSNR values can be obtained from the below formulas for an image.

- Mean Square Error (MSE): It is the squared difference between the original and the denoised image. This gives us the difference between original image and the denoised image. It can be calculated as shown in Equation.1

$$
M S E=\frac{1}{M N} \sum_{m=0}^{M-1} \sum_{n=0}^{N-1}[y(m, n)-y(m, n)]^{2}
$$

where $y(m, n)$ is the original image and $y^{`}(m, n)$ is denoised image with relation to image dimension $(m, n)$.

- Root Mean Square Error (RMSE): It is the square root of the MSE value obtained using Equation. 1 and can be calculated using Equation 2.

$$
R M S E=\sqrt{M S E}
$$

- Peak signal to noise ratio (PSNR): It is the ratio between maximum possible power of a signal and the power of corrupting noise that affects the quality and reliability of its representation. PSNR is calculated as shown in Equation.3

$$
\text { PSNR }=10 \log _{10}\left(\frac{\text { MAX }^{2}}{\text { MSE }^{2}}\right)
$$


Where MSE is mean square error and MAX is the maximum pixel value of image.

Table 2, gives the values obtained for preprocessing the $\mathrm{X}$-Ray images considered.

Table 2. Values Obtained during Filtering Process Considering X-ray Images

\begin{tabular}{|c|r|c|c|r|r|r|r|r|r|}
\cline { 2 - 10 } \multicolumn{1}{c|}{} & \multicolumn{9}{c|}{ Filtering } \\
\cline { 2 - 11 } \multicolumn{1}{c|}{} & \multicolumn{3}{c|}{ Weiner } & \multicolumn{3}{c|}{ Mean } & \multicolumn{3}{c|}{ Median } \\
\hline Images & \multicolumn{1}{c|}{ MSE } & RMSE & PSNR & \multicolumn{1}{c|}{ MSE } & RMSE & PSNR & \multicolumn{1}{c|}{ MSE } & RMSE & PSNR \\
\hline 1 & 8.3923 & 2.8977 & 38.9232 & 28.5731 & 5.3454 & 33.6052 & 21.6735 & 4.6555 & 34.8055 \\
\hline 2 & 28.3825 & 5.3234 & 33.6343 & 76.5692 & 8.7504 & 29.3235 & 42.2153 & 6.4973 & 31.9101 \\
\hline 3 & 13.1362 & 3.6244 & 36.9802 & 34.1822 & 5.8465 & 32.8268 & 12.1298 & 3.4828 & 37.3263 \\
\hline 4 & 14.9598 & 3.8678 & 36.4155 & 49.4437 & 7.0319 & 31.2234 & 35.6401 & 5.9699 & 32.6454 \\
\hline 5 & 9.6559 & 3.1074 & 38.3169 & 28.4969 & 5.3382 & 33.6168 & 9.9051 & 3.1472 & 32.2062 \\
\hline 6 & 12.4835 & 3.5332 & 37.2014 & 39.8002 & 6.3087 & 32.1659 & 13.8036 & 3.7153 & 36.7649 \\
\hline 7 & 4.3441 & 2.0843 & 41.7858 & 7.7353 & 2.7812 & 39.2845 & 3.3595 & 1.8329 & 32.902 \\
\hline 8 & 14.2912 & 3.7804 & 36.6141 & 48.2108 & 6.9434 & 31.3334 & 34.5843 & 5.8808 & 32.776 \\
\hline Avg & & & & & & & & & \\
PSNR & & & $\mathbf{3 7 . 4 8 3 9}$ & & & $\mathbf{3 2 . 9 2 1 4 4}$ & & & $\mathbf{3 3 . 9 1 7 0 5}$ \\
Value & & & & & & & & & \\
\hline
\end{tabular}

In a similar manner, values of other modality- CT, MRI, Bone scan images are obtained.

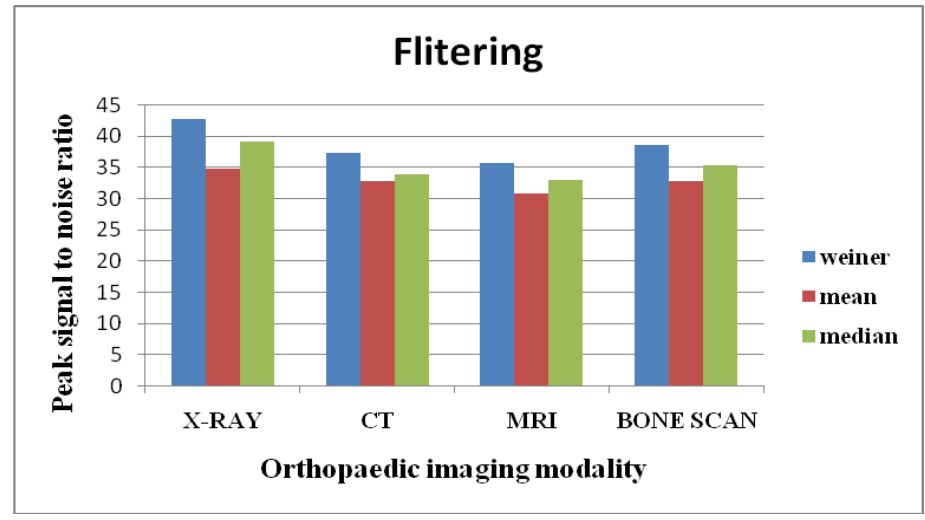

Figure 7. PSNR Values Considering Different Filters

From the values given in Table 2, average values are drawn (x-ray modality only), PSNR values considering all the modalities taken for the work are as shown in Figure 7. With reference to pre-processing stage, we conclude that weiner filter gave highest PSNR and hence all the images considered for the further stages, will be processed by weiner filtering.

2.0.3. Wavelets Features: 8 We have used wavelets to identify the higher and lower frequency components of the images. The advantage of inherent denoising capability of wavelets is taken and the images are processed. Since the X-Ray, CT scan, MRI and Bone scan images are more exposed to varying frequency radiations; wavelets help to identify these variations efficiently than any other mathematical transformations.

Discrete Wavelet Transform (DWT) is chosen for analysis because of the wavelets inherent multiresolution properties and it preserves high and low frequency feature therefore preserving peaks and valleys found in typical spectra. DWT separates the fine 
scale and large scale information in the original image into the wavelet detail and approximation co-efficients respectively and the wavelet co-efficients includes all the information of the original image. Wavelet family, namely, haar, daubechies, symlets and coiflets are used for the work.

Wavelets approximation is obtained from decomposing the images using the above said wavelets. For these approximations, features, namely, mean, standard deviation; median, variance and entropy are computed using Equation (4), (5), (6).

Mean $=(1 / 2)\left[\sum \sum \mathrm{iP}[\mathrm{i}, \mathrm{j}]+\sum \sum \mathrm{jP}[\mathrm{i}, \mathrm{j}]\right]$

i j $\quad$ i j

Variance $=(1 / 2)\left[\sum \sum(\mathrm{i}-\mu)^{2} \mathrm{P}[\mathrm{i}, \mathrm{j}]+\sum \sum(\mathrm{j}-\mu)^{2} \mathrm{P}[\mathrm{i}, \mathrm{j}]\right]$

$$
\text { i j i j }
$$

Entropy $=-\sum \sum \mathrm{P}[\mathrm{i}, \mathrm{j}] \times \log \mathrm{P}[\mathrm{i}, \mathrm{j}]$

$$
\text { i j }
$$

Where $\mathrm{P}$ is an image. We have named the features individually as given in Table 3.

Table 3. Indiviual Features with their Abbreviation

\begin{tabular}{|c|c|c|}
\hline SI no & Abbreviation & Feature \\
\hline 1 & F1 & Mean \\
\hline 2 & F2 & Standard deviation \\
\hline 3 & F3 & Median \\
\hline 4 & F4 & Variance \\
\hline 5 & F5 & Entropy \\
\hline
\end{tabular}

2.0.4. Level Selection: Pre-processed images are used to extract the features for experimentation. Wavelets were applied to decompose the images into various sub-bands. For the proposed work, we have applied five level of decomposition. Table 4, shows the values extracted for first level of decomposition. To show the workflow, values are drawn for only 1st level of decomposition using Haar wavelet transform. In similar manner, we have done for four wavelets, namely, haar, daubechies, symlets and coiflets.

Figure 8, gives the performance of classification for Haar wavelet at different levels with different combination of images for training, validation and testing such as $70 \%$ of images are used training, $15 \%$ of images are used validation and $15 \%$ of images are used testing. Similarly, 60\%-20\%-20\% and 50\%-25\%-25\% and 30\%-35\%-35\%. Also, from Figure 8 , we observe that 3 rd level of decomposition gave better results for the images considered. 4th and 5th level decomposition performance was found to be decreasing; hence 3rd level was considered for further experimentation.

Table 4. Feature Values of Haar Wavelet at 1st Level of Decomposition

\begin{tabular}{|c|c|c|c|c|c|r|}
\cline { 2 - 7 } \multicolumn{2}{c|}{} & \multicolumn{5}{c|}{ Features } \\
\hline Modality & Images & F1 & F2 & F3 & F4 & \multicolumn{1}{|c|}{ 55 } \\
\hline \multirow{4}{*}{$\begin{array}{c}\text { Bone } \\
\text { Scan }\end{array}$} & 1 & 316.4346 & $5.19 \mathrm{E}+01$ & $3.29 \mathrm{E}+02$ & 2695.743 & $2.36 \mathrm{E}-02$ \\
\cline { 2 - 7 } & 2 & 330.9125 & $3.43 \mathrm{E}+01$ & $3.42 \mathrm{E}+02$ & 1173.212 & 0 \\
\cline { 2 - 7 } & 3 & 292.2512 & $4.90 \mathrm{E}+01$ & $3.05 \mathrm{E}+02$ & 2403.679 & 0 \\
\cline { 2 - 7 } & 4 & 308.7498 & $4.03 \mathrm{E}+01$ & $3.24 \mathrm{E}+02$ & 1621.508 & 0 \\
\cline { 2 - 7 } & 5 & 307.9439 & $4.86 \mathrm{E}+01$ & $3.25 \mathrm{E}+02$ & 2358.473 & 0 \\
\cline { 2 - 7 } & 6 & 316.4346 & $5.19 \mathrm{E}+01$ & $3.29 \mathrm{E}+02$ & 2695.743 & $2.36 \mathrm{E}-02$ \\
\hline
\end{tabular}




\begin{tabular}{|c|c|c|c|c|c|c|}
\hline & 7 & 333.9261 & $4.73 E+01$ & $3.59 \mathrm{E}+02$ & 2240.085 & $3.90 \mathrm{E}-03$ \\
\hline & 8 & 327.5162 & $6.71 \mathrm{E}+01$ & $3.58 \mathrm{E}+02$ & 4498.521 & 0 \\
\hline \multirow{8}{*}{ CT } & 1 & 44.20737 & $6.47 \mathrm{E}+01$ & 0 & 4186.73 & $1.04 \mathrm{E}+00$ \\
\hline & 2 & 102.5787 & $5.79 \mathrm{E}+01$ & $1.07 \mathrm{E}+02$ & 3352.666 & $5.58 \mathrm{E}-01$ \\
\hline & 3 & 142.4562 & $3.76 \mathrm{E}+01$ & $1.34 \mathrm{E}+02$ & 1412.58 & $2.82 \mathrm{E}-02$ \\
\hline & 4 & 60.13127 & $7.45 \mathrm{E}+01$ & $2.12 \mathrm{E}+00$ & 5548.786 & $1.06 \mathrm{E}+00$ \\
\hline & 5 & 143.5055 & $8.32 \mathrm{E}+01$ & $1.43 \mathrm{E}+02$ & 6915.265 & $1.79 \mathrm{E}-01$ \\
\hline & 6 & 144.1584 & $8.08 \mathrm{E}+01$ & $1.53 \mathrm{E}+02$ & 6522.791 & $2.44 \mathrm{E}-01$ \\
\hline & 7 & 64.45371 & $4.12 \mathrm{E}+01$ & $7.07 \mathrm{E}+01$ & 1693.414 & $7.97 \mathrm{E}-01$ \\
\hline & 8 & 52.41617 & $6.62 \mathrm{E}+01$ & 0 & 4377.062 & $1.06 \mathrm{E}+00$ \\
\hline \multirow{8}{*}{ MRI } & 1 & 143.6544 & $8.36 \mathrm{E}+01$ & $1.37 \mathrm{E}+02$ & 6989.769 & 0 \\
\hline & 2 & 76.91942 & $9.27 \mathrm{E}+01$ & $4.24 \mathrm{E}+01$ & 8587.632 & $1.00 \mathrm{E}+00$ \\
\hline & 3 & 136.6034 & $1.19 \mathrm{E}+02$ & $1.12 \mathrm{E}+02$ & 14062.58 & $8.31 \mathrm{E}-01$ \\
\hline & 4 & 127.6306 & $1.21 \mathrm{E}+02$ & $1.03 \mathrm{E}+02$ & 14535.52 & $9.51 \mathrm{E}-01$ \\
\hline & 5 & 119.664 & $7.47 \mathrm{E}+01$ & $1.09 \mathrm{E}+02$ & 5576.393 & $8.17 \mathrm{E}-03$ \\
\hline & 6 & 90.02537 & $9.87 \mathrm{E}+01$ & $5.59 \mathrm{E}+01$ & 9750.383 & $9.79 \mathrm{E}-01$ \\
\hline & 7 & 72.4368 & $6.51 \mathrm{E}+01$ & $7.14 \mathrm{E}+01$ & 4242.603 & $8.26 \mathrm{E}-01$ \\
\hline & 8 & 91.15808 & $8.93 \mathrm{E}+01$ & $6.79 \mathrm{E}+01$ & 7973.822 & $9.33 \mathrm{E}-01$ \\
\hline \multirow{8}{*}{ X-Ray } & 1 & 90.64473 & $6.56 \mathrm{E}+01$ & $8.77 \mathrm{E}+01$ & 4308.94 & $3.65 \mathrm{E}-01$ \\
\hline & 2 & 93.9514 & $9.17 \mathrm{E}+01$ & $6.65 \mathrm{E}+01$ & 8401.543 & $1.99 \mathrm{E}-01$ \\
\hline & 3 & 104.3806 & $9.43 \mathrm{E}+01$ & $7.64 \mathrm{E}+01$ & 8889.876 & $1.89 \mathrm{E}-03$ \\
\hline & 4 & 151.3796 & $1.03 \mathrm{E}+02$ & $1.90 \mathrm{E}+02$ & 10642.91 & $1.13 \mathrm{E}-01$ \\
\hline & 5 & 79.75499 & $8.86 \mathrm{E}+01$ & $2.97 \mathrm{E}+01$ & 7853.652 & $1.01 \mathrm{E}-01$ \\
\hline & 6 & 116.8894 & $9.25 \mathrm{E}+01$ & $1.37 \mathrm{E}+02$ & 8561.34 & $8.45 \mathrm{E}-01$ \\
\hline & 7 & 116.8894 & $9.25 \mathrm{E}+01$ & $1.37 \mathrm{E}+02$ & 8561.34 & $8.45 \mathrm{E}-01$ \\
\hline & 8 & 104.0671 & $9.62 \mathrm{E}+01$ & $9.83 \mathrm{E}+01$ & 9248.298 & $8.48 \mathrm{E}-01$ \\
\hline
\end{tabular}

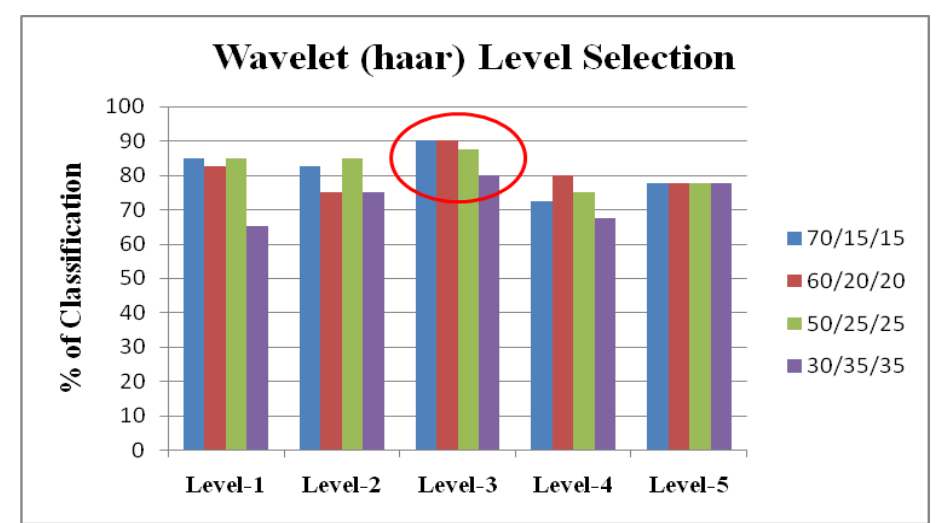

Figure 8. Classification Performance Considering Haar Wavelet for all the Levels

2.0.5. Feature Reduction: Initially five features, namely, like mean, standard deviation, median, variance and entropy are computed to form the feature set. Table 5, gives the values obtained using haar wavelet at 3rd level of decomposition; values are shown considering only eight images to understand the workflow easily. From Figure 9, we observe that the classification accuracy is low for F1-mean and F5-entropy. Mean gives the contribution of individual pixel intensity for the entire image as intensity is varying in all the modalities considered \& entropy is a statistical measure of randomness as randomness is more in Bone scan images and more over two totally different images can have same mean and entropy which degraded the performance. We have tried combination of features, namely, two, three. 


\section{Table 5. Values Obtained using Haar Wavelet at 3rd Level of Decomposition}

\begin{tabular}{|c|r|r|r|r|}
\hline Images & \multicolumn{1}{|c|}{ Bone Scan } & \multicolumn{1}{c|}{ CT } & \multicolumn{1}{c|}{ MRI } & \multicolumn{1}{c|}{ X-Ray } \\
\hline 1 & 632.86924 & 88.41473 & 287.3087 & 181.2895 \\
\hline 2 & 661.82493 & 205.1574 & 153.8388 & 187.9028 \\
\hline 3 & 584.50235 & 284.9125 & 273.2069 & 208.7612 \\
\hline 4 & 617.49968 & 120.2625 & 255.2611 & 302.7593 \\
\hline 5 & 615.88778 & 287.0109 & 239.328 & 159.51 \\
\hline 6 & 632.86924 & 288.3169 & 180.0507 & 233.7788 \\
\hline 7 & 667.8523 & 128.9074 & 144.8736 & 233.7788 \\
\hline 8 & 655.03249 & 104.8323 & 182.3162 & 208.1341 \\
\hline
\end{tabular}

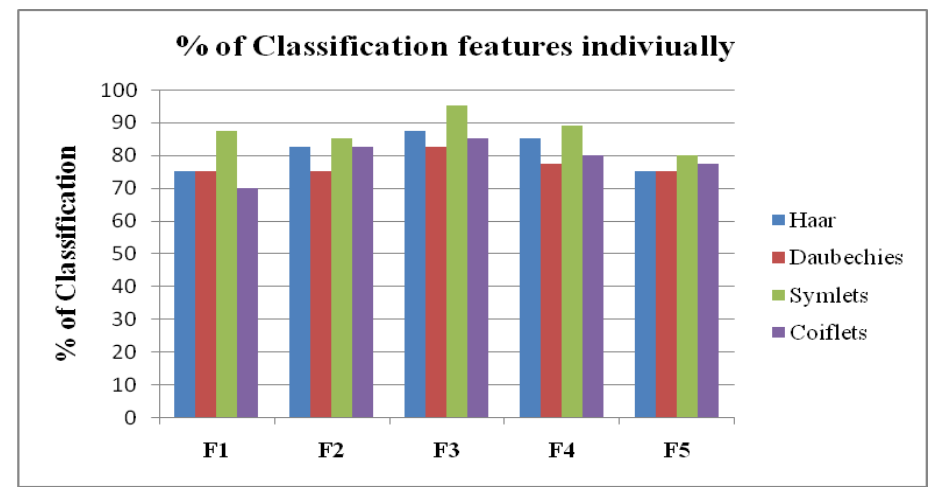

\section{Figure 9. Performance of Classification for Four Wavelets Considering Indiviual Features}

We have considered the combined feature Fij, where $i, j=1$ to 5 and $i \neq j$. The feature F12 means combination of Energy and Mean features. Similarly, the feature F123 means combination of Energy, Mean and Standard deviation, and so on. However, combination of features in groups of two, three and four for four wavelets i.e., Haar, Daubechies, Symlets and coiflets wavelet are studied.

Certain combinations of features are discarded as the percentage of classification is only in the range [50\% - $60 \%$ ] and even below. The features discarded are F14, F15, F125, F12, F123, and F1235. These are discarded as all the combination invariably contain either F1 or F5 which gave poor performance when considered individually also. Thus, we have arrived at the 2 best features used to classify orthopedic imaging modality, namely, F23 and F234 as shown in the Figure 10.

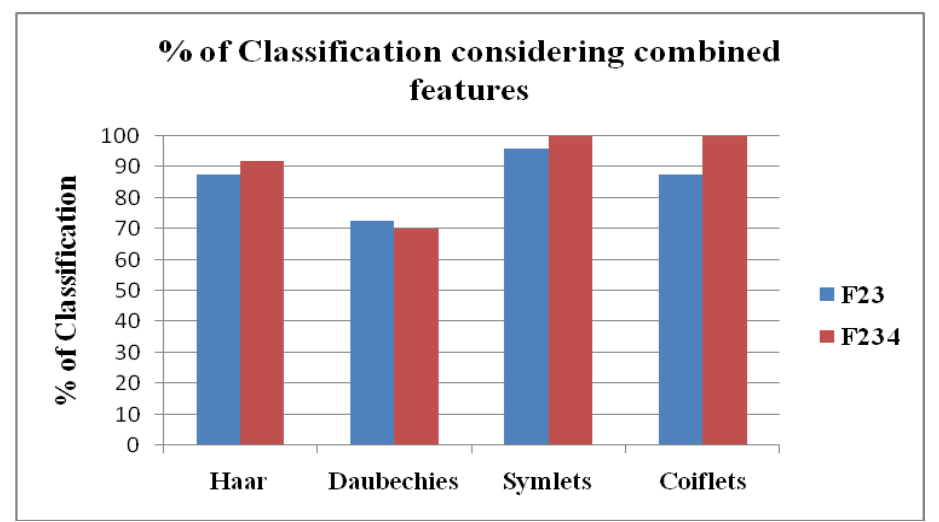

Figure 10. Performance of Classification of Four Wavelets for Selected Features 
2.0.6. Classifier: ANN classifier is a better option for problems with scope for approximation. The computed feature values for the current problem exhibit slight variations. Hence the ANN classifier is chosen. The six features extracted based on the co-efficients obtained from wavelet families, are input to the neural network with five input nodes. The four output nodes correspond to the four bit output vector identifying the orthopedic imaging modality as X-ray, CT, MRI and Bone scan. The hidden layer contains eight nodes. The neural network is trained using back propagation-learning algorithm. The stabilized weights are reloaded and test vectors are input during testing. The optimal number of hidden layer neurons is chosen using the criterion given in Equation (7).

$n=c \sqrt{\frac{N}{d \log N}}$

Where, $\mathrm{n}=$ number of hidden layer neurons, $\mathrm{C}=$ constant yielding optimal performance, $\mathrm{d}=$ number of features, and $\mathrm{N}=$ number of rows in the training sample matrix.

Validation set is carried out to design the ANN for optimal performance. The ANN exhibits optimal performance for minimum mean squared error (MSE). The MSE is plotted with varying number of hidden neurons. Figure 11 shows the validation MSE is used to decide the number of nodes in the hidden layer. Since the minimum MSE is observed when 8 nodes are used in the hidden layer, the hidden layer of the designed ANN contains 8 nodes.

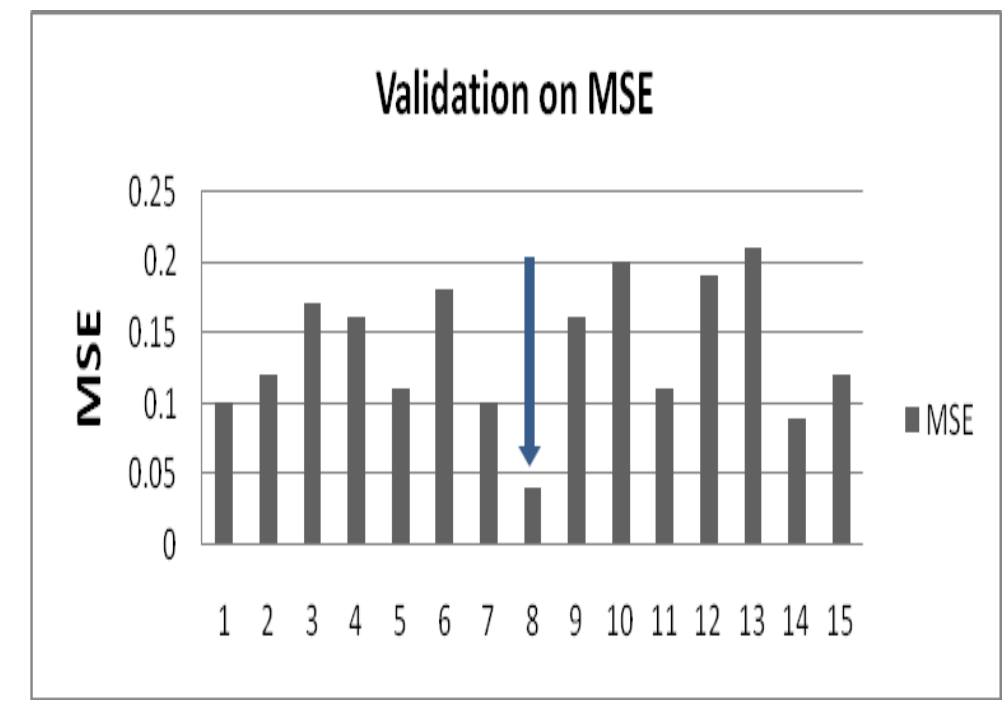

Figure 11. Validation MSE for Varying Number of Hidden Layer Neurons

The details of different parameters are set on trial basis and the values used are as given in Table 6. 
Table 6: Parameters of Neural Network

\begin{tabular}{|c|c|}
\hline PARAMETERS & VALUES \\
\hline \multicolumn{2}{|l|}{ Input layer } \\
\hline No of input neurons & 5 ,depending on (Feature reduction) \\
\hline \multicolumn{2}{|l|}{ Hidden layer } \\
\hline No of neurons & 8 \\
\hline Transfer/activation function & TRAINGDA \\
\hline \multicolumn{2}{|l|}{ Output layer } \\
\hline No of neurons & 4(equal to number of output classes) \\
\hline Learning constant & 0.01 \\
\hline Acceptable error(MSE) & 0.00001 \\
\hline Number of epochs & 1000 \\
\hline Termination conditions & $\begin{array}{l}\text { Based on minimum Mean square } \\
\text { error(MSE) or maximum number of epochs } \\
\text { allowed }\end{array}$ \\
\hline
\end{tabular}

In the identification process, the output of neural network is represented by a vector of four binary values. Each binary value is associated with unique modality. For example, vector [1000] represents X-Ray modality. The output vectors representing different types of bones are given in Table.7.

Table 7. Output Vectors

\begin{tabular}{|c|l|}
\hline Output & \multicolumn{1}{|c|}{ Type } \\
\hline 1000 & X-ray \\
\hline 0100 & CT \\
\hline 0010 & MRI \\
\hline 0001 & Bone scan \\
\hline
\end{tabular}

\section{Results and Discussion}

Orthopedic imaging modality, namely, X-Ray, CT, MRI and Bone scan images are considered. The database of images contains a total of 800 images which includes 200 for each modality. The database is divided into three subsets: training, validation and testing with randomly chosen samples. Table 8 shows the results of classification for various combinations of training, validation and testing sets with ANN classifier. The classification accuracies are nearly $100 \%$ for the used feature sets. The classification performance decreases with increase in the size of feature set. The performance of the built neural network is analyzed for different combinations of training, validation and testing sets. 


\section{Table 8. Classification for Various Combinations of Training, Validation and Testing}

\begin{tabular}{|c|c|c|c|c|c|c|c|}
\hline \multicolumn{3}{|c|}{ No of images } & \multicolumn{3}{l}{ Combined results of classification for training, validation and testing(Total of } \\
\hline \multirow{2}{*}{ Training } & Validation & Testing & True positive & False negative & True negative & False positive & Accuracy \\
\hline & & & & & & \\
\hline 240 & 280 & 280 & 368 & 56 & 372 & 28 & 0.925 \\
\hline 280 & 240 & 280 & 360 & 76 & 344 & 28 & 0.88 \\
\hline 280 & 280 & 240 & 320 & 76 & 364 & 20 & 0.855 \\
\hline 320 & 200 & 280 & 344 & 64 & 264 & 28 & 0.76 \\
\hline 320 & 240 & 240 & 312 & 56 & 360 & 20 & 0.84 \\
\hline 320 & 280 & 200 & 264 & 56 & 312 & 20 & 0.72 \\
\hline 360 & 160 & 280 & 328 & 104 & 320 & 60 & 0.81 \\
\hline 360 & 200 & 240 & 352 & 92 & 344 & 52 & 0.87 \\
\hline 360 & 240 & 200 & 392 & 108 & 352 & 44 & 0.93 \\
\hline 360 & 280 & 160 & 312 & 88 & 312 & 36 & 0.78 \\
\hline 400 & 160 & 240 & 336 & 96 & 320 & 28 & 0.82 \\
\hline 400 & 200 & 200 & 304 & 76 & 340 & 40 & 0.805 \\
\hline 400 & 240 & 160 & 288 & 68 & 360 & 20 & 0.81 \\
\hline 440 & 160 & 200 & 312 & 60 & 348 & 28 & 0.825 \\
\hline 440 & 200 & 160 & 312 & 76 & 336 & 52 & 0.81 \\
\hline 480 & 160 & 160 & 352 & 88 & 332 & 52 & 0.855 \\
\hline 480 & 120 & 200 & 312 & 92 & 304 & 28 & 0.77 \\
\hline 480 & 200 & 120 & 304 & 100 & 296 & 60 & 0.75 \\
\hline 520 & 160 & 120 & 280 & 76 & 328 & 28 & 0.76 \\
\hline 520 & 120 & 160 & 296 & 100 & 352 & 36 & 0.81 \\
\hline
\end{tabular}

From the Table 9, the average classification accuracy is over $97 \%$ for the databases of size 200 to 400 images; hence the approach delivers a second opinion to orthopeadicians reliably.

\section{Table 9. Classification Accuracy for Varying Size of Data Base.}

\begin{tabular}{|c|c|c|c|c|c|c|c|c|c|c|c|c|c|c|c|c|}
\hline $\begin{array}{l}\text { Classification } \\
\text { accuracy }\end{array}$ & \multicolumn{16}{|c|}{ Size of the database } \\
\hline Size of the & \multicolumn{4}{|c|}{ Images $=200$} & \multicolumn{4}{|c|}{ Images $=400$} & \multicolumn{4}{|c|}{ Images $=600$} & \multicolumn{4}{|c|}{ Images $=800$} \\
\hline Modality* & $\mathbf{A}$ & B & C & D & A & B & C & D & $\mathbf{A}$ & B & $\mathbf{C}$ & D & $\mathbf{A}$ & B & $\mathbf{C}$ & D \\
\hline Minimum & 90 & 96 & 97 & 96 & 88.1 & 93 & 90 & 89 & 74 & 81 & 82 & 75 & 67 & 74 & 70 & 61.4 \\
\hline Maximum & 97 & 100 & 100 & 98 & 90.2 & 95 & 96 & 92 & 86.5 & 85.8 & 86.3 & 80 & 72.4 & 80 & 78 & 64 \\
\hline Average & 93.5 & 98 & 98.5 & 97 & 89.1 & 94 & 93 & 90.5 & 80.2 & 83.4 & 84.15 & 77.5 & 69.7 & 77 & 74 & 62.7 \\
\hline
\end{tabular}




\section{Conclusions}

We conclude that combination of two, three and four groups of features has given good results compared with individual features. Feature F23 and F234 with symlet wavelet transform gave highest accuracy considering 200 images and decreased with increase in data set. An average classification accuracy of $98 \%$ is seen for all the modalities considered. The work is useful in automatic recognition of modality before images are interpreted in the area of orthopaedics.

\section{References}

[1] S. Pittner and S. V. Kamarthi, "Feature extraction from wavelet coefficients for pattern recognition tasks", in IEEE Transactions on Pattern Analysis and Machine Intelligence, vol. 21, no. 1, (1999), pp. 83-88.

[2] G. Ding, Q. Dai, W. Xu and F. Yang, “Affine-Invariant Image Retrieval Based on Wavelet Interest Points", IEEE 7th Workshop on Multimedia Signal Processing, Shanghai, (2005), pp. 1-4. 2005

[3] K. Wang, Z. Ren and X. Xiong, "Combination of Wavelet and SIFT Features for Image Classification Using Trained Gaussion Mixture Model”, Intelligent Information Hiding and Multimedia Signal Processing, (2008), pp. 79-82.

[4] S. Arivazhagan, R. A. Priyadharshini and S. Seedhanadevi, "Object recognition based on gabor wavelet features", Devices, Circuits and Systems (ICDCS), (2012), pp. 340-344.

[5] Zhang Feng, Liu Shang-qian, Wang Da-bao and Guan Wei, "Aircraft recognition in infrared image using wavelet moment invariants", Image and Vision Computing (2009), pp. 313-318.

[6] Dinggang Shen, Horace H.S Discriminative wavelet shape descriptors for recognition of 2-D patterns, Pattern Recognition, vol. 32, no 2, (1999), pp. 151-165.

[7] T. Chang and C. C. J. Kuo, "Texture analysis and classification with tree-structured wavelet transform", in IEEE Transactions on Image Processing, vol. 2, no. 4, (1993), pp. 429-441.

[8] J. Gllavata, R. Ewerth and B. Freisleben, "Text detection in images based on unsupervised classification of high-frequency wavelet coefficients", 17th International Conference on Pattern Recognition, vol. 1, (2004), pp. 425-428.

[9] C. Busch, "Wavelet based texture segmentation of multi-modal tomograpic images", Computers \& Graphics, vol. 21, no 3, (1997), pp. 347-358.

[10] S. Arivazhagan and L. Ganesan, "Texture segmentation using wavelet transform", Pattern Recognition Letters, vol. 24, (2003), pp. 3197-3203.

[11] Malik and T. Zremic, "Classification of medical images using energy information obtained from wavelet transform for medical image retrieval", Proceedings of 7th International Workshop on Enterprise networking and computing in Healthcare Industry, (2005), pp. 124-129.

[12] Mojsilovic and J. Gomes, "Semantic based categorization, browsing and retrieval in medical image databases", International Conference on Image Processing, vol. 3, (2002). pp. III-145-III-148.

[13] P. Y. Lau and S. Ozawa, "Image classification for different imaging modalities in image-guided medical diagnosis model", IEEE EMBS Asian-Pacific Conference on Biomedical Engineering, (2003), pp. 150151.

[14] G. Csurka, S. Clinchant and G. Jacquet, "Medical image modality classification and retrieval", 9th International Workshop on Content-Based Multimedia Indexing, (2011), pp. 193-198.

[15] H. Pourghassem and H. Ghassemian, "Content-based medical image classification using a new hierarchical merging scheme”, Computing Medical Imaging Graph, vol. 32, no 8, (2008), pp. 651-61.

[16] D. Keysers, J. Dahmen, H. Ney, B. B. Wein and T. M. Lehmann, "Statistical frame work for model based image retrieval in medical applications", J Electronic Image, vol. 12, no 1, (2003), pp. 59-68.

[17] M. M. Rahman, P. Bhattacharya and B. C. Desai, "A frame work for medical image retrieval using machine learning and statistical similarity matching techniques with relevance feedback", IEEE Trans. Information Technology, vol. 11, no 1, (2007), pp. 58-69.

[18] H. Greenspan and A. T. Pinhas, "Medical image categorization and retrieval for PACS using the GMMKL frame work", IEEE Transactions on Information Technology, vol. 11, no. 2, (2007), pp. 190-202.

[19] P. Tirilly, K. Lu, X. Mu, T. Zhao and Y. Cao, "On modality classification and its use in text-based image retrieval in medical databases", IEEE workshop on Content-Based Multimedia Indexing (CBMI), (2011), pp. 13-15.

[20] U. Avni, "X-ray image categorization and retrieval using patch based visual words representation", IEEE Symposium, vol. 1, no. 2, (2009), pp. 350-353.

[21] G. Quellec, M. Lamard, G. Cazuguel, B. Cochener and C. Roux, "Wavelet optimization for contentbased image retrieval in medical databases", Medical Image Analysis, vol. 14, (2010), pp. 227-241. 